\title{
Alterations of glutathione and GSTM1 mutations induce tumor metastasis and invasion via EMT pathway in breast cancer patients
}

Arshad Ali ( $\nabla$ arshadali@mail.nwpu.edu.cn )

Northwestern Polytechnical University School of Life Sciences https://orcid.org/0000-0002-4076-6620

Ayaz Ali

Pir Mehr Ali Shah Arid Agriculture University: University of Arid Agriculture

Shafiq Ahmad

Kohat University of Science and Technology

\section{Research Article}

Keywords: Glutathione, Glutathione S-Transferase M1, Metastasis, Invasion, Proliferation, Breast cancer

Posted Date: May 12th, 2021

DOl: https://doi.org/10.21203/rs.3.rs-515612/v1

License: (a) (i) This work is licensed under a Creative Commons Attribution 4.0 International License. Read Full License 


\section{Abstract}

Purpose: Alteration in the Glutathione (GSH) and Glutathione S-Transferase (GST) family lead to various disorders including breast cancer. However, the role of GSH and GSTM1 in the onset of breast cancer is still not fully elucidated.

Objective: In the present study we observed considerable deficiency in the levels of glutathione and genetic mutation in the GSTM1 enzyme that influence susceptibility to breast cancer metastasis and invasion via EMT pathway.

Methods: GSTM1 genotype was identified by multiplex polymerase chain reaction (PCR), RT-PCR and western blotting in breast cancer tissue samples and ANCT samples. The endogenous glutathione levels were determined by HPLC. The tumor metastasis, invasion and EMT biomarkers were determined by RT-PCR and western blot. The relationship between breast cancer, disease progression and histological status were estimated by one way analysis of variance and descriptive statistic. Data were analyzed using OriginPro 2015 statistics software (OriginLab, Northampton, USA). The correlation among different factors was assessed at $95 \%$ confidence intervals $(\mathrm{Cl})$ using the Mann-Whitney, Kruskal Wallis, and ANOVA test. $\mathrm{P}<0.05$ was considered significant.

Results: In present study genotyping analysis of GST investigated that genetic mutation in GSTM1 was detected in breast cancer tissue samples. Moreover, messenger RNA and protein analysis showed that GSTM1 was significant downregulated in tumor tissues $(p=0.005, p=0.02)$ of breast cancer patients. Furthermore, significant reduction in the level of total glutathione level $(G S H t P<0.05)$ was observed among correlation with patient ages, stages and histological grades, of breast cancer patients. Additionally, the result revealed that downregulation of GSTM1 promotes EMT pathway that leads to enhanced the expression of tumor proliferation, invasion and metastasis in breast cancer tissue samples compared with the ANCT samples $(P<0.05)$.

Conclusions The present findings suggest that GSTM1 genotype could be a potential biomarker that regulate EMT pathway associated with breast cancer prognosis.

\section{Introduction}

Breast cancer is most frequently diagnosed cancer among women and is the second leading cause of cancer death in females' worldwide. It is initiated in the breast tissues possesses milk producing glands known as lobules and ducts connecting lobules to the nipple, whereas the other parts are composed of fatty, connective and lymphatic tissues. The onset of breast cancer occurs in both sexes but are comparatively rare in males [1]. Generally, breast cancer accounts for $23 \%$ of all cancer cases and $14 \%$ cancer death have been considered, and their incidence rate are usually higher in Western and European countries [2].

Glutathione, a tripeptide composed of cysteine, glycine, and glutamic acid, provides protection against ROS either directly as an antioxidant agent or indirectly, by supporting other cellular antioxidants in a working state [3]. Glutathione (GSH) plays central role in a number of cellular processes such as differentiation, proliferation and apoptosis and aberration in GSH homeostasis are contributing in the progression and 
development of different human diseases including cancer. GSH form a complexes with a different variety of product of oxidative stress and carcinogens via reactions promoted by the enzyme glutathione Stransferase (GST). An equilibrium GSH: GSSG ratio is important for survival of the cells and control regulation of the system is therefore very necessary. Alteration in GSH or GSH activity has also been studied in the trabecular mesh work and aqueous humor of patients with glaucoma [4]. Glutathione (GSH) and glutathione-S-transferases (GSTs) are important first lines of defence system against both acute and chronic toxicities of electrophiles and reactive oxygen/nitrogen species. Both GSH concentrations and GST enzyme functions are under equilibrium homeostatic control. Glutathione S-Transferase (GST) conjugate with glutathione and confer anti-oxidant protection through neutralization of toxic carbonyl, epoxide, and peroxide containing metabolites produced within the cell by oxidative stress [5]. GSTs are responsible for substantial proportion of total glutathione peroxidase activity in humans [6].

GST family genes plays a critical role in various biochemical and molecular aspects of different cellular resistance process. Metabolic detoxification is one of the basic mechanism of cellular resistance govern by GST family [7]. The GST family include GSTT1, GSTM1 and GSTP1 genes that encode most essential phase II detoxifying enzymes involved in the conjugation of substrates that are toxic to cancer cells, including anthracyclines as a chemotherapeutic agents used in breast cancer treatment. Both GSTT and GSTM1 classes posses polymorphic null forms (GSTT1 null and GSTM1 null), which lack their two alleles and are therefore unable to encode the detoxifying enzymes [8]. Thereof, homozygous deletion of glutathione S-transferase M1 (GSTM1), results in accumulation of oxidative stress byproducts which determines its protective role against antioxidant as well [9]. Reduction in GSTT1 and GSTM1 activities closely associated with increased risk of developing different cancers [10]. Numerous studies reveal the association of null genotype with increased risk of developing breast cancer [11]. According to meta analysis, GSTM1 null genotype significantly increased the risk of breast cancer (1:10) ratios varies in different ethnicity, such as Asian (1:21) and Caucasian (1:05) especially in postmenopausal woman (1:11) [12]. Furthermore, D. N. Chirilã et al investigate that null genotype of GSTM1 is a potential risk factor to develop synchronous breast cancers and for breast cancer linked with one extra mammary cancer. The presence of GSTM1 and GSTT1 null genotype lead to increased risk for multiple breast cancer (bilateral or synchronous) [11]. A functional role of GSTM1 was determined in vascular smooth muscle cells (VSMC), showing that decreased expression of GSTM1 results to increased oxidative stress, cell migration and proliferation [13]. In breast cancer, genetic polymorphisms of GSTT1, GSTM1, and GSTP1 may be involved in modifying the response to neoadjuvant chemotherapy, given that null alleles or the alteration in the genes expression resulting to enzyme deficiency, which is associated with the inability of the chemotherapy drug to export cells [14]. However, the association of GSTM1 within Pakistani breast cancer populations have not been investigated so far. The present study attempts to elucidate the role GSTM1 in breast cancer tissues and to highlight it possible role in regulation of EMT pathway that associated with breast cancer pathogenesis, which may be used as early prognosis biomarker of breast cancer in clinical settings.

\section{Materials And Methods}

\section{Subject enrollment and sample collection}


Tumor tissue samples were collected from one hundred and ninety eight breast cancer patients and adjacent normal control tissue (ANCT) samples in RNAlater ${ }^{\circledR}$ stabilization solution (Thermo Fisher, Waltham, USA) at the time of surgery from different hospital at Khyber Pakhtunkhwa, Pakistan. ANCT was selected from the area about $2 \mathrm{~cm}$ away from the affected site by an oncologist on the basis of histopathological observations. Patients with history of other infectious and familial diseases were excluded from this study. This study was conducted after prior approval from the Ethical Review Committee of the collaborating hospital. Prior to samples collection, written informed consent was obtained from patients participated in the study and the collected samples were stored at $-80^{\circ} \mathrm{C}$ for further analysis.

\section{DNA Extraction, gel electrophoresis, and quantification}

DNA was extracted from the breast cancer tissue samples and ANCT samples using the standard phenolchloroform method [15]. PCR was performed to determined the genotype of GSTM1. The PCR products were electrophoresis using $2 \%$ agarose gel. Thereafter, stained gel with ethidium bromide and visualized under the UV illuminator BioDoc Analyze TM (Biometra, Göttingen, Germany). The bands intensities were quantified using ImageJ densitometer (University of Wisconsin, Madison, USA).

\section{Total RNA Extraction, cDNA synthesis and expression Analysis}

Real time RT-PCR was performed according to previous literature. Total RNA was extracted from breast cancer tissue samples and ANCT samples using total RNA kit 1 (OMEGA) and reversely transcribed into complementary DNA (cDNA) using commercially available cDNA reverse transcriptase kit (TAKARA). Then, qPCR detection of gene expression was performed with specific primers and SYBR Green using GAPDH as an internal control. The thermal cycling conditions included initial denaturation step at $95^{\circ} \mathrm{C}$ for $30 \mathrm{~s}, 40$ cycles at $95^{\circ} \mathrm{C}$ for $10 \mathrm{~s}, 60^{\circ} \mathrm{C}$ for $20 \mathrm{~s}, 72^{\circ} \mathrm{C}$ for $5 \mathrm{~s}$. Gene specific primers for respective genes (sense primer) and (antisence primer) were design. Then RT- PCR were performed to analyzed the expression of target genes in breast cancer tissue samples and ANCT samples according to manufacture protocol. Data will be analyzed by $2^{-\Delta \Delta C t}$ method.

\section{Protein extraction and western blot analysis}

Western blot was performed in tissue lysates using GAPDH as an internal control. Briefly, total proteins were extracted from breast cancer tissues samples and ANCT samples in lysis buffer. Proteins concentrations were determined using BCA method and then proteins were resolved on 8-10\% SDS-PAGE and consequently transferred to a PVDF membrane. The membrane was incubated in $5 \%$ skim milk for $1 \mathrm{~h}$. Then membrane was incubated using primary antibodies for overnight at $4^{\circ} \mathrm{C}$. Rabbit polyclonal antibodies were used as primary antibodies after diluted in 1X TBST. The membranes were washed three times followed by incubation with secondary antibodies for $2 \mathrm{~h}$ at room temperature. Finally, measure the blots using the ECL kit according to the manufacturer's protocols.

\section{HPLC separation, quantification and determination of GST subunits and activity}


All the procedure were performed at $4^{\circ} \mathrm{C}$. The tumors tissues and ANCT tissues (weights ranging from 35-65 $\mathrm{mg}$ ) were homogenized in $3.0 \mathrm{ml}$ of Tris/ $\mathrm{HCl}(25 \mathrm{mM}, \mathrm{pH} 7.4)$, using an ultra-turrax. To reduced contamination of the connective tissues, epithelium layer from the cystadenomas were removed from the cyst wall and used for additional analysis. For cytosolic preparation, homogenized samples were centrifuged at highest speed for 85 minutes. Cytosolic glutathione was purified following the Bogaards et al., 1989 [16] protocol. The equilibration and condition of column were performed in 5 minutes obtaining the initial conditions. $200 \mathrm{ul}$ of the sample extract and the standards were injected into the chromatographic column maintained at $35^{\circ} \mathrm{C}$. In brief, selected amount of cytosol was used to a $2 \mathrm{ml} \mathrm{S}$-hexylgluthathioneagarose affinity column, wash with $16 \mathrm{ml}$ buffer containing $0.4 \mathrm{M} \mathrm{NaCl}$ solution, and eluted in the same buffer solution containing $5 \mathrm{mM}$ of S-hexylglutathione. Elutes precursory of the S-hexylglutathione alpha were analyzed for total glutathione activity, and mostly less than $5 \%$ of the total applied enzymatic activity were present. The concentration of elutes were changed to approximately $0.2 \mathrm{ml}$ with the help of centricon PM 10 ultra filtration tube. The glutathione subunits were eluted with incline of acetonitrile in water, both including $0.1 \%$ trifluoroacetic acid (from 40 to $50 \%$ acetonitrile in $18 \mathrm{~min}$, proceeded by an additional increase to $53 \%$ in $5 \mathrm{~min}$ and isocrating separation for another $7 \mathrm{~min}$ ). The detection of elution profile was observed by measuring the absorbance at $214 \mathrm{~nm}$.

\section{Data analysis}

Data were analyzed using OriginPro 2015 statistics software (OriginLab, Northampton, USA). The correlation among different factors was assessed at $95 \%$ confidence intervals $(\mathrm{Cl})$ using the Mann-Whitney, Kruskal Wallis, and ANOVA test. Specific comparison were undertaken by standard descriptive analysis, and p-value of $<0.05$ was considered significant.

\section{Results}

\section{Genotyping determination of GSTM1 in breast cancer patients}

The distribution of GSTM1 genotype in the breast cancer tissues and ANCT tissues were determined by standard PCR as shown in figure $1 \mathrm{~A}$ and table 1 . The frequency of null GSTM1 genotype was significantly higher (0.733) in breast cancer tissues samples compared with the ANCT samples $(0.134)(p=0.004)$, Further, we categorized the breast cancers patients based on their age, it was revealed that the null-GSTM1 genotype frequency was significantly higher in patients with age less than $50(<50)$ years as compared with patients of greater than $50(>50)$ years age of patients $(0.705 \mathrm{vs} 0.582, p=0.007)$. These data indicate that mutation in GSTM1 genotype has significant association with early development of breast cancer in Pakistani population. In addition, HPLC result determined that the level of endogenous glutathione (GSHt, GSH, GSSG) were decreased in patients with age less than $50(<50)$ years compared with patients having age greater than $50(>50)$ years as shown in figure 2 .

\section{Determination of GSTM1 expression in breast cancer patients}

Western blotting and RT-PCR were performed to evaluate the protein and mRNA expression levels of GSTM1 in both tumor and control individual tissues. The relative expression of GSTM1 mRNA transcript was found 
to be significantly decreased $(p=0.001)$ in the tumor tissues samples compared to the ANCT samples, irrespective of the histological and disease status of the patient (Figure 1B). Furthermore, the concentration of GSTM1 proteins were determined by western blot. The result concluded that the protein concentration of GSTM1 was significantly decreased in breast cancer tissue samples $(p=0.003)$ as compared with ANCT samples as shown in figure 1C. These result revealed that downregulation of GSTM1 genotype promotes EMT pathway that enhanced the expression of tumor metastasis and invasion biomarkers which is mainly associated with the development and progression of breast carcinomas.

\section{Determination of GSH concentration among patients with different age groups}

Glutathione (GSH) plays crucial role in a multitude of cellular processes, including cell proliferation, differentiation, and apoptosis and alteration in GSH homeostasis are participating in the development and progression of different diseases, including cancer. The deficiency of GSH or decrease in the ratio of GSH/glutathione disulphide (GSSG) leads to enhanced susceptibility to oxidative stress implied in the development of cancer. Therefore, the endogenous GSH (GSHt, GSH, GSSG) concentrations were determined by HPLC and compared between two study cohorts of breast cancer, $<45$ and $>45$ years. Interestingly, in both study cohorts, the GSHt concentration was higher in the control tissues (135.206 \pm 39.031 and $138.59 \pm 38.438$ respectively) compared to that of tumor tissues $(22.478 \pm 4.792$ and $30.468 \pm 5.758$ respectively). Statistical analysis showed that GSHt concentrations were significantly associated with both tissue types ( $p=0.003, p=0.005)$, while GSH and GSSG concentrations were also decreased in tumor tissues of both study cohorts, but no significant difference was observed $(p>0.05)$ between tumor tissue samples and ANCT samples (Figure 3, S Table 1). These data indicated that endogenous GSH, especially GSHt was significantly downregulated in tumor tissues in both study cohorts.

\section{GSH concentration with premenopausal and postmenopausal status of breast cancer patients}

Further, the endogenous concentration of GSH were investigated among premenopausal and postmenopausal breast cancer patients. Of the total study cohorts $(n=198), 115$ belonged to the premenopausal group and 83 belonged to the postmenopausal group. The GSHt concentration was significantly lower in the tumor tissues samples of premenopausal group $(25.52 \pm 4.361)$ compared to ANCT samples $(133.479 \pm 32.372, p=0.006)$. Similarly, significant reduction in GSHt was observed in the tumor tissues samples of postmenopausal group (30.602 \pm 7.422$)$ compared with the ANCT samples (145.183 $\pm 51.330, p=0.009)$. In addition, the concentration of GSH and GSSG were also significantly decreased in premenopausal tumor tissues samples $(p<0.05)$, while no difference has been observed in postmenopausal tumor tissues samples $(p>0.05)$ as compared with the ANCT samples. The data obtained suggests that GSHt was significantly downregulated in the tumor tissues samples of both premenopausal and postmenopausal patients (Figure 4, S Table 2).

\section{Determination of GSH among different stages and grades in breast cancer patients}

Moreover, the endogenous GSH levels were determined in different stages and grades of breast cancer. According to stages wise stratified, 86 samples belong to stage I, 64 belong to stage II and 48 belong to stage III of the study subjects as shown in table. The data reveal that the GSHt levels were decreased in 
breast cancer tissue samples in all tumor stages (I, II and III) $(24.499 \pm 24.527,29.838 \pm 7.459$, $28.556 \pm 14.428)$ compared to ANCT samples $(134.955 \pm 34.488,110.811 \pm 41.883,136.776 \pm 96.714)$. The results obtained were statistically more significant in stages I and II of breast cancer $(p=0.014, p=0.003)$ as that of stage III in breast cancer patients $(p=0.031)$. The concentration of GSH, and GSSG was decreased as advancement in stages of breast cancer, however, the results were statistically not significant $(p>0.05)$ (Figure 5A, S Table 3). Further, their levels were also correlated with different grades of breast cancers. Of total study cohorts $(n=198), 78$ grade I, 63 grade II, and 57 individuals belong to grade III. Similarly, the levels of GSHt were higher in all grades of tumor tissues samples $(211.675 \pm 122.27,128.452 \pm 29.468$, $142.11 \pm 82.047)$ compared to ANCT samples $(19.013 \pm 7.762,26.19 \pm 4.194,26.798 \pm 11.984)$. These results were also more significant in grades I and II of breast cancer patients $(p=0.03, p=0.036)$ as compared to grade III individuals $(p=0.05)$. Hence, the concentration of GSH and GSSG has no significant correlation with tumor grades (Figure 5B, S Table 4).

\section{Downregulation of GSTM1 promotes tumor proliferation, metastasis and invasion in breast cancer patients}

Present study further evaluate the expression of various biomarkers, including tumor, proliferation, and invasion and metastasis in the cancer tissue samples and ANCT samples of breast cancer patients. The mRNA expression showed that tumor biomarkers, including HER2 and CEA were significantly upregulated in tumor tissues ( $p=0.001, p=0.03$ ) as compared with ANCT. Interestingly, upregulation of HER2 expression is more significant than CEA (Figure 6A). Similarly, tumor proliferation biomarkers were also analyzed in breast cancer tissues using RT- PCR. The mRNA expression revealed that proliferation markers, including Ki-67, PAKT, and Bcl2 were significantly increased in tumor tissues samples compared to ANCT samples $(p=0.006$, $p=0.009, p=0.014$ ) (Figure 6B). The result was highly significant and show that the tumor tissues has high rate of proliferation. For further validation, tumor metastasis and invasion were determined in the tumor tissue samples and ANCT samples. The results revealed that the expression of metastasis and invasion biomarkers, including MMP-2 and MMP-9 were significantly increased in breast cancer tissue samples verses ANCT samples $(p=0.007, p=0.009)$ (Figure $6 \mathrm{C}$ ).

\section{Downregulation of GSTM1 induces Epithelial to mesenchymal cell transition pathway}

Epithelial to mesenchymal cell transition (EMT) plays a crucial role in appropriate morphogenesis during development. Disregulation of this process has been concerned as a key events in fibrosis and the progression of carcinomas to a metastatic state. However we analyzed EMT process in breast cancer tissue samples and ANCT samples. Both RNA and proteins were isolated to determined EMT pathway through RTpcr and western blot. We finalized that downregulation of GSTM1 induced the mRNA expression of mesenchymal marker i.e vimentin $(* p=0.019)$ Snail $(* * p=0.0074)$ and Fibronectin $(* * p=0.0089)$ via decreased the expression of epithelial marker i.e E-cadherin ( $\left.{ }^{* *} p=0.009,\right)$, MUC-1 $\left({ }^{*} p=0.0082\right)$ and lamine $(* * p=0.0097)$ in breast cancer tissue samples as compared to their ANCT samples. In addition, Present study also determined that the protein expression of mesenchymal markers vimentin, snail were increased (** $\left.p=0.006,{ }^{* *} p=0.0027\right)$ while the expression of epithelial marker i.e E-cadherin, MUC-1 were decreased (** $=0.0057,{ }^{* *} p=0.0078$ ) in breast cancer tissue samples as compared to ANCT samples using GAPDH as negative control as shown in figure 7 . Each results were repeated as triplicate. 


\section{Discussion}

Several studies indicate that oxidative stress has been involved in the development and progression of cancer, clarifying that antioxidant treatment may contribute in protection from cancers [17]. Oxidative stress mostly implicated by a malignant cell is greater than their respective benign counterpart. Glutathione (GSH) plays central role in a number of cellular processes, such as differentiation, proliferation, and apoptosis. Aberration in GSH homeostasis are contributing in the progression and development of numerous human diseases, including cancer. The deficiency of GSH or reduction in the ratio of GSH/glutathione disulphide (GSSG) leads to promote susceptibility to oxidative stress intimated in the development of cancer. The depletion in GSH or blocking of GST results to enhanced cytotoxicity via oxidative stress in melanoma cells $[18,19]$.

In the current study, the levels GSHt, GSH and GSSG were decreased according to the patients age, histological status and tumor stages and grades. Thereof, the concentration GSH total were significantly correlated with premenopausal status of patients. High levels of oxidative stress and decreased antioxidant status is strongly associated with age of individual in most of population [20]. The following studied also support our hypothesis that the GSH concentration is decreased in patient with age less than $50(<50)$ as shown in Fig. 2. Previous study concluded that the amount of GSSG produced as a results of reaction among GSH and ROS is quick revert back to normal GSH, thus stabilizing the normal redox cycle.

In normal situations GSSG plays key role, to stable GSH in its reduced form [21]. Our results also supports the hypothesis that GSH levels were significantly decreased in breast cancer patients compared with control subjects, determining the mechanism that protection against ROS controlled by GSH could be lowered in breast cancer patients, possibly due to a abnormal redox cycle. The intracellular oxidation and redox level is well be balanced in the cells. The alteration in the redox level would cross this balance and occupied the potential to promote the oxidation level [22]. Didziapetriene $\mathrm{J}$, et al [23] investigated that the antioxidant pathways and especially GSH levels are downregulated in breast cancer tissues Reduction in the ratios of GSH to GSSG were investigated in both serum and tissue samples of breast cancer patients as compared to serum and ANCT samples of the same patients. Our results are in agreement with already reported studies that the GSH and GSSG levels were decreased in breast cancer patients [23].

In current study, we also determined the possible association of GSTM1 genetic polymorphism with development of breast cancer. The GSTs enzymes play important role in the detoxification of different products implied by cancer therapy, excited us to determined the prognostic efficiency of GSTs genotype deletion in breast cancer. Several studies have investigating the role of deletion in GST genotyping in correspondence with the development of breast cancers but still the results are conflicting [24]. Our finding support the previous result that the GSTM1 genotype was deleted in the tumor tissue samples of breast patients as shown in Fig. 1A.

The GSTM1 null genotype has been closely associated with the development of brain, stomach and lung cancer $[25,26])$. With regard to breast carcinoma, the GSTM1 null genotype has not been closely linked with the elevated risk but revealed as a high risk factor for the eldest postmenopausal women also supported the present hypothesis. Vaury et al [27] investigated that the GSTM1 genotype is involved to provoke CYPJA1 
gene transcription. The deletion in GSTM1 and GSTT1 alone or in combination with CYPIA1 polymorphisms leads to develop breast cancer. The deletion of both alleles of the GSTM1 gene is exist in $30-60 \%$ of mostly population, leading in complete deficit of the concerned enzyme [28]. In addition another study determined that GSTM1 null genotype or CYPJA1 polymorphism might be linked to increased the risk of breast cancer in postmenopausal Caucasian women [29].

Revival of the EMT process is commonly regarded to be a core component of tumor progression and development. EMT is an embryonic network that relaxed cell-cell adhesion complexes and increased cells invasiveness and migration. EMT signaling pathway has also been considered as therapeutic targets in different disease, considering in a breast cancer in vitro models murine pancreatic cancer model, and even in clinical settings [30]. EMT is the course in which breast cancer cells lose epithelial features and gain mesenchymal phenotypes. Therefore, the present study determined the expression of EMT associated proteins in breast cancer tissue samples. Consistent with the reported results, we also found that the expression of epithelial markers was decreased, while the expression of mesenchymal markers were increased in breast cancer tissue samples, suggesting that GSTM1 might regulate the growth and aggressiveness of breast cancer through EMT process. A significant role of GSTM1 was determined in vascular smooth muscle cells (VSMC), identified that the downregulation of GSTM1 expression in these cells leads to enhanced oxidative stress, cell migration, metastasis and proliferation [13]. These studies support our results that the expression of tumor proliferation, metastasis and invasion biomarker is upregulated via EMT pathway in breast cancers tissues.

\section{Conclusion And Future Perspective}

In conclusion, our outcomes identified that GSTM1 was downregulated in breast cancer tissues, and contributed to invasion, metastasis and migration abilities of breast cancer. Moreover, low expression of GSTM1 led to reduced the level of total GSH and mainly associated with poor prognosis in breast cancer patients. Simultaneously, the implementation of GSTM1 role was also correlated with the expression of proliferation, metastasis and invasion as well as EMT-related biomarkers in breast cancer tissues. These findings suggested new insights into the regulatory mechanism of GSTM1 in breast cancer. A limitation of our study was the lack of in vitro experiments to confirm the findings on breast cancer cells, and the lack of specific signaling pathway involved in GSTM1 regulation of breast cancer proliferation and apoptosis. Additional studies need to further elucidated the role GSTM1 in lymph node metastasis. In the next plan, we will focus on solving these problems.

\section{Declarations}

\section{Conflict of Interest}

Authors declare that they have no competing interests.

\section{Author disclosures and declarations}


There is no-financial interests, This study was conducted after prior approval from the Ethical Review Committee of the collaborating hospital. Prior to samples collection, written informed consent was obtained from patients participated in the study,

\section{Acknowledgments}

The authors specially acknowledge and are thankful to all the participants as well as the supporting staff at district head quarter hospitals, KPK, Pakistan for their kind cooperation and provision of the Breast tissue samples. There is no funding for this study.

\section{Author Contributions}

Conception: Arshad Ali

Interpretation or Analysis of Data: Ayaz Ali and Shafiq Ahmad

Preparation of the manuscript: Arshad Ali and Shafiq Ahmad

Revision for important intellectual content: Ayaz Ali and Arshad Ali

Supervision: Arshad Ali

\section{References}

1. Malik FA, Sanders AJ, Jones AD, Mansel RE, Jiang WG. Transcriptional and translational modulation of KAl1 expression in ductal carcinoma of the breast and the prognostic significance. Int J Mol Med. 2009;23(2):273-8.

2. Ward E, Jemal A, Thun M. Regarding "Increase in breast cancer incidence in middle-aged women during the 1990s". Ann Epidemiol. 2005;15(6):424-5. author reply 426-427.

3. Gherghel D, Griffiths HR, Hilton EJ, Cunliffe IA, Hosking SL. Systemic reduction in glutathione levels occurs in patients with primary open-angle glaucoma. Invest Ophthalmol Vis Sci. 2005;46(3):877-83.

4. Ferreira SM, Lerner SF, Brunzini R, Evelson PA, Llesuy SF. Oxidative stress markers in aqueous humor of glaucoma patients. Am J Ophthalmol. 2004;137(1):62-9.

5. Strange RC, Spiteri MA, Ramachandran S, Fryer AA. Glutathione-S-transferase family of enzymes. Mutat Res. 2001;482(1-2):21-6.

6. Huang J, Tan PH, Tan BK, Bay BH. GST-pi expression correlates with oxidative stress and apoptosis in breast cancer. Oncol Rep. 2004;12(4):921-5.

7. The diabetes prevention. program: evaluation and management of diabetes. Response to Adler and Turner and Singer et al The DPP Research Group. Diabetes Care. 1999;22(10):1757-8.

8. Mcllwain CC, Townsend DM, Tew KD. Glutathione S-transferase polymorphisms: cancer incidence and therapy. Oncogene. 2006;25(11):1639-48. 
9. Suvakov S, Damjanovic T, Stefanovic A, Pekmezovic T, Savic-Radojevic A, Pljesa-Ercegovac M, Matic M, Djukic T, Coric V, Jakovljevic J, et al. Glutathione S-transferase A1, M1, P1 and T1 null or low-activity genotypes are associated with enhanced oxidative damage among haemodialysis patients. Nephrol Dial Transplant. 2013;28(1):202-12.

10. Rebbeck TR. Molecular epidemiology of the human glutathione S-transferase genotypes GSTM1 and GSTT1 in cancer susceptibility. Cancer Epidemiol Biomarkers Prev. 1997;6(9):733-43.

11. Chirila DN, Balacescu O, Popp R, Oprea A, Constantea NA, Vesa S, Ciuce C. GSTM1, GSTT1 and GSTP1 in patients with multiple breast cancers and breast cancer in association with another type of cancer. Chirurgia (Bucur). 2014;109(5):626-33.

12. Qiu LX, Yuan H, Yu KD, Mao C, Chen B, Zhan P, Xue K, Zhang J, Hu XC. Glutathione S-transferase M1 polymorphism and breast cancer susceptibility: a meta-analysis involving 46,281 subjects. Breast Cancer Res Treat. 2010;121(3):703-8.

13. Yang Y, Parsons KK, Chi L, Malakauskas SM, Le TH. Glutathione S-transferase-micro1 regulates vascular smooth muscle cell proliferation, migration, and oxidative stress. Hypertension. 2009;54(6):1360-8.

14. Mishra A, Chandra R, Mehrotra PK, Bajpai P, Agrawal D. Glutathione S-transferase M1 and T1 polymorphism and response to neoadjuvant chemotherapy (CAF) in breast cancer patients. Surg Today. 2011;41(4):471-6.

15. Strauss WM: Preparation of genomic DNA from mammalian tissue. Curr Protoc Neurosci 2001, Appendix 1:Appendix 1H.

16. Bogaards JJ, van Ommen B, van Bladeren PJ. An improved method for the separation and quantification of glutathione S-transferase subunits in rat tissue using high-performance liquid chromatography. J Chromatogr. 1989;474(2):435-40.

17. Cabello CM, Bair WB 3rd, Wondrak GT. Experimental therapeutics: targeting the redox Achilles heel of cancer. Curr Opin Investig Drugs. 2007;8(12):1022-37.

18. Traverso N, Ricciarelli R, Nitti M, Marengo B, Furfaro AL, Pronzato MA, Marinari UM, Domenicotti C. Role of glutathione in cancer progression and chemoresistance. Oxid Med Cell Longev. 2013;2013:972913.

19. Benlloch M, Ortega A, Ferrer P, Segarra R, Obrador E, Asensi M, Carretero J, Estrela JM. Acceleration of glutathione efflux and inhibition of gamma-glutamyltranspeptidase sensitize metastatic B16 melanoma cells to endothelium-induced cytotoxicity. J Biol Chem. 2005;280(8):6950-9.

20. Knight JA. The biochemistry of aging. Adv Clin Chem. 2000;35:1-62.

21. DeLeve LD, Kaplowitz N. Glutathione metabolism and its role in hepatotoxicity. Pharmacol Ther. 1991;52(3):287-305.

22. Liang H, Zhou Z, Luo R, Sang M, Liu B, Sun M, Qu W, Feng F, Liu W. Tumor-specific activated photodynamic therapy with an oxidation-regulated strategy for enhancing anti-tumor efficacy. Theranostics. 2018;8(18):5059-71.

23. Didziapetriene J, Kazbariene B, Tikuisis R, Dulskas A, Dabkeviciene D, Lukoseviciene V, Kontrimaviciute E, Suziedelis K, Ostapenko V. Oxidant/Antioxidant Status of Breast Cancer Patients in Pre- and PostOperative Periods. Medicina (Kaunas) 2020, 56(2). 
24. Gudmundsdottir K, Tryggvadottir L, Eyfjord JE. GSTM1, GSTT1, and GSTP1 genotypes in relation to breast cancer risk and frequency of mutations in the p53 gene. Cancer Epidemiol Biomarkers Prev. 2001;10(11):1169-73.

25. Kano SI, Choi EY, Dohi E, Agarwal S, Chang DJ, Wilson AM, Lo BD, Rose IVL, Gonzalez S, Imai T, et al: Glutathione S-transferases promote proinflammatory astrocyte-microglia communication during brain inflammation. Sci Signal 2019, 12(569).

26. Chen ZH, Xian JF, Luo LP. Association between GSTM1, GSTT1, and GSTP1 polymorphisms and gastric cancer risk, and their interactions with environmental factors. Genet Mol Res 2017, 16(1).

27. Vaury C, Laine R, Noguiez P, de Coppet P, Jaulin C, Praz F, Pompon D, Amor-Gueret M. Human glutathione S-transferase M1 null genotype is associated with a high inducibility of cytochrome P450 1A1 gene transcription. Cancer Res. 1995;55(23):5520-3.

28. Pacholak LM, Amarante MK, Guembarovski RL, Watanabe MAE, Panis C. Polymorphisms in GSTT1 and GSTM1 genes as possible risk factors for susceptibility to breast cancer development and their influence in chemotherapy response: a systematic review. Mol Biol Rep. 2020;47(7):5495-501.

29. Bailey LR, Roodi N, Verrier CS, Yee CJ, Dupont WD, Parl FF. Breast cancer and CYPIA1, GSTM1, and GSTT1 polymorphisms: evidence of a lack of association in Caucasians and African Americans. Cancer Res. 1998;58(1):65-70.

30. Marcucci F, Stassi G, De Maria R. Epithelial-mesenchymal transition: a new target in anticancer drug discovery. Nat Rev Drug Discov. 2016;15(5):311-25.

\section{Tables}

Table 1: Analysis of GST genotyping in breast cancer patients and it correlation with different of

\begin{tabular}{|c|c|c|c|c|c|c|c|c|c|c|c|c|}
\hline \multirow[t]{2}{*}{ Genotype } & \multicolumn{3}{|c|}{$\begin{array}{l}\text { Tumor tissues } \\
\text { (198) }\end{array}$} & \multicolumn{2}{|c|}{ ANCT (198) } & \multicolumn{4}{|c|}{$\begin{array}{l}\text { Greater than } 50 \\
\text { year }(86)\end{array}$} & \multicolumn{3}{|c|}{$\begin{array}{l}\text { Less than } 50 \text { year } \\
\text { (112) }\end{array}$} \\
\hline & & $\mathrm{N}$ & $\bar{F}$ & $\bar{N}$ & $\mathrm{~F}$ & $?$ & $\mathrm{~N}$ & $\bar{F}$ & $\mathrm{P}$ & $\mathrm{N}$ & $\mathrm{F}$ & $\mathrm{P}$ \\
\hline \multirow[t]{2}{*}{ GSTM1 } & wild & 53 & $(0.267)$ & 169 & $(0.866)$ & 0.004 & 36 & $(0.418)$ & NS & 33 & $(0.295)$ & 0.007 \\
\hline & Null & 145 & $(0.733)$ & 29 & $(0.134)$ & & 50 & $(0.582)$ & & 79 & $(0.705)$ & \\
\hline \multirow[t]{2}{*}{ GSTT1 } & Wild & 135 & $(0.681)$ & 146 & $(0.737)$ & NS & 51 & $(0.593)$ & NS & 72 & $(0.643)$ & NS \\
\hline & Null & 63 & $(0.319)$ & 52 & $(0.263)$ & & 35 & $(0.407)$ & & 40 & $(0.357)$ & \\
\hline \multirow[t]{2}{*}{ GSTP1 } & Wild & 139 & $(0.702)$ & 161 & $(0.813)$ & NS & 56 & $(0.651)$ & NS & 78 & $(0.696)$ & NS \\
\hline & Null & 59 & $(0.298)$ & 37 & $(0.187)$ & & 30 & $(0.349)$ & & 34 & $(0.304)$ & \\
\hline \multirow[t]{2}{*}{ GSTM1+GSTT1 } & wild & 138 & $(0.696)$ & 162 & $(0.818)$ & NS & 54 & $(0.627)$ & NS & 81 & $(0.723)$ & NS \\
\hline & Null & 60 & $(0.304)$ & 36 & $(0.182)$ & & 32 & $(0.737)$ & & 31 & $(0.277)$ & \\
\hline
\end{tabular}


GSTM1= glutathione S-transferase Mu, GSTT1= glutathione S-transferase theta, GSTP1 = glutathione S-transferase pi, ANCT = Adjacent normal control tissue, $\mathrm{N}=$ number, $\mathrm{F}=$ frequency, $\mathrm{P}=\mathrm{P}$ value,

\section{Figures}

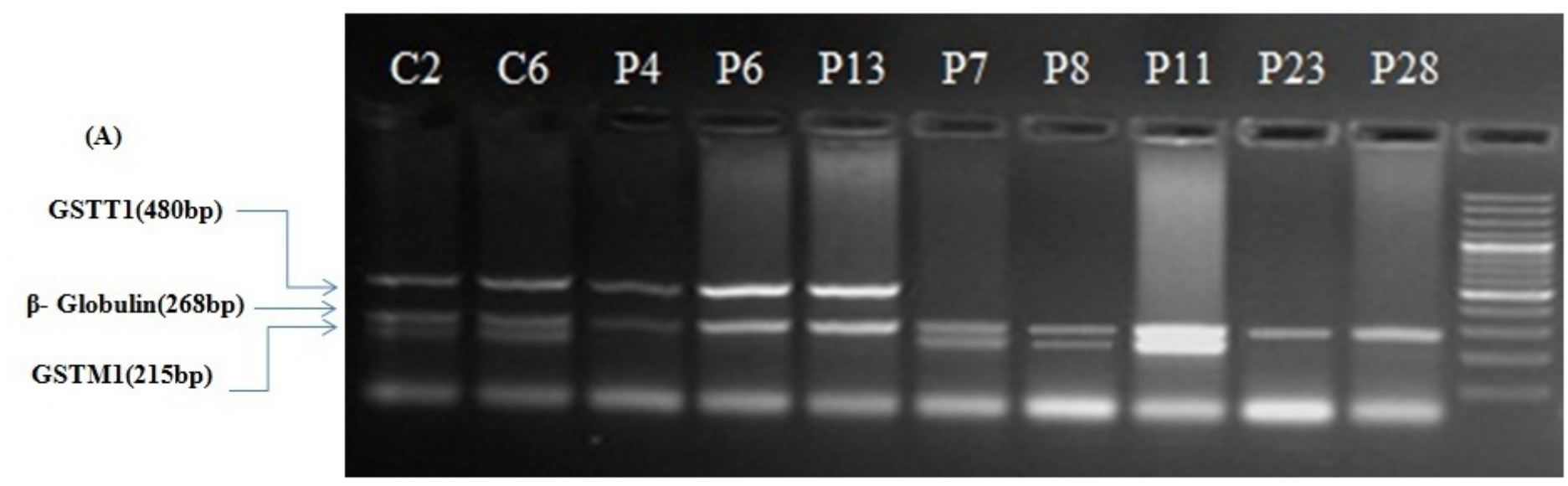

(B)

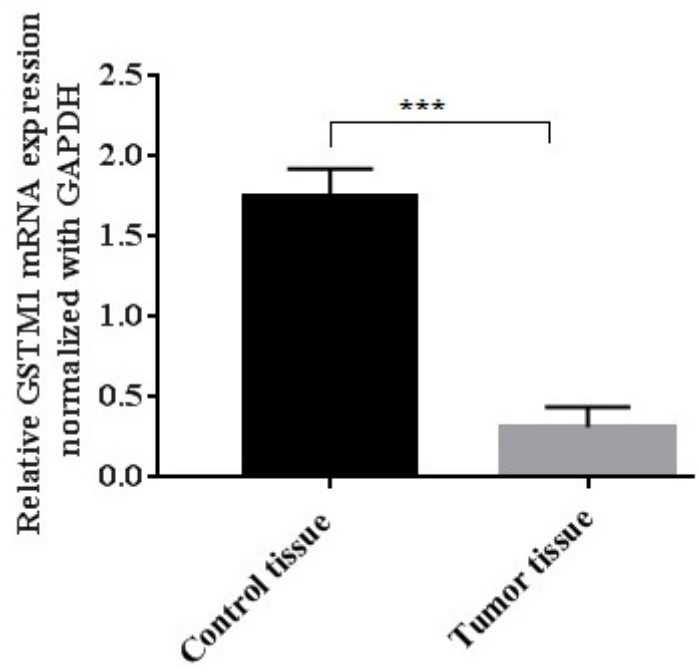

(C)

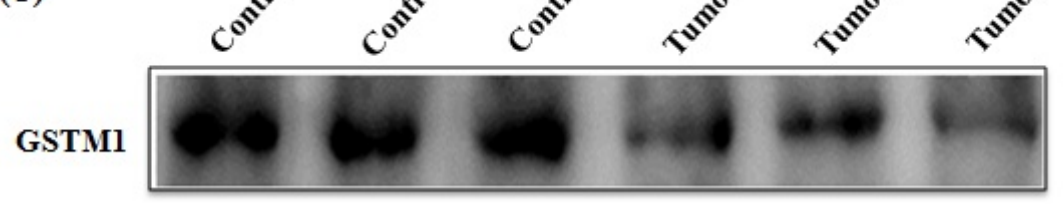

GAPDH

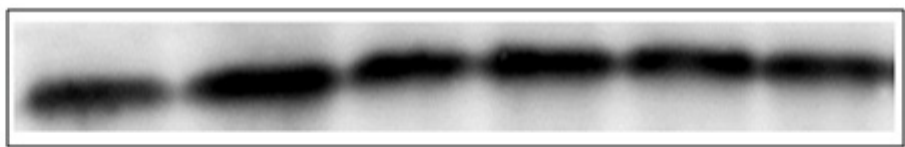

Figure 1

Genotyping determination of GST in breast tumor tissue samples compared with ANCT samples (A) PCR identification of GSTM1 genotype (B) mRNA expression of GSTM1 ( $* \star * P=0.001)$ (C) Protein expression of GSTM1 compared with GAPDH as a internal control in breast cancer patients. 


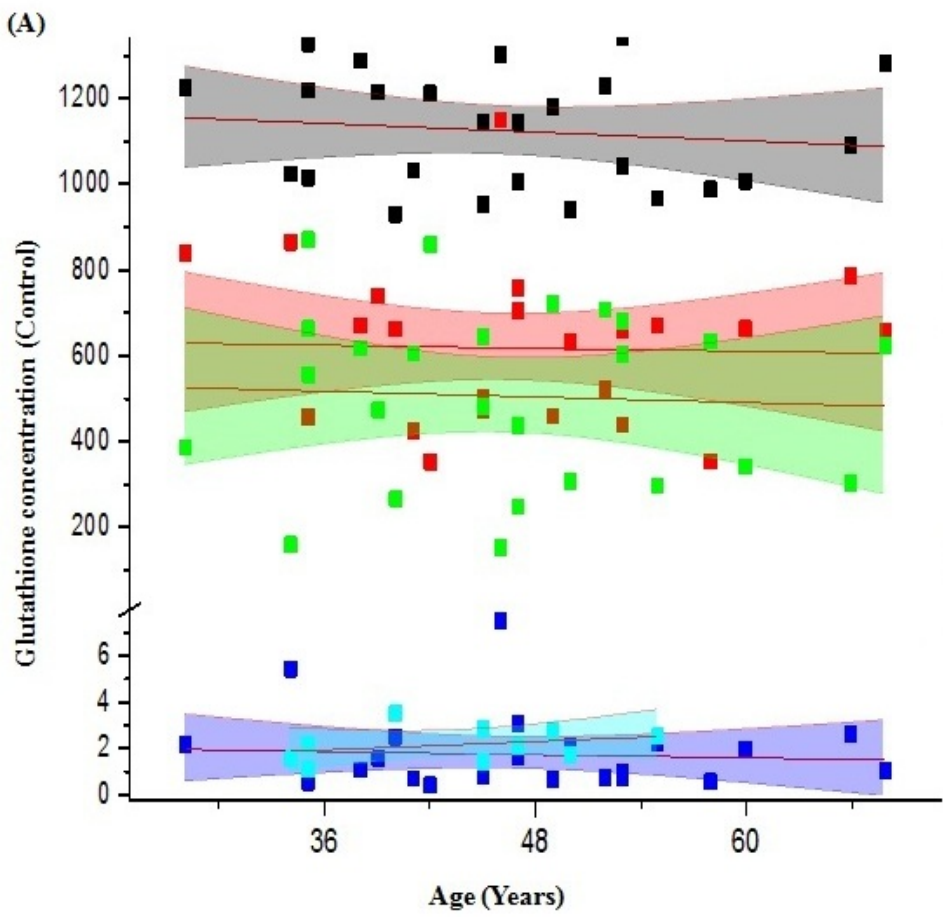

(B)

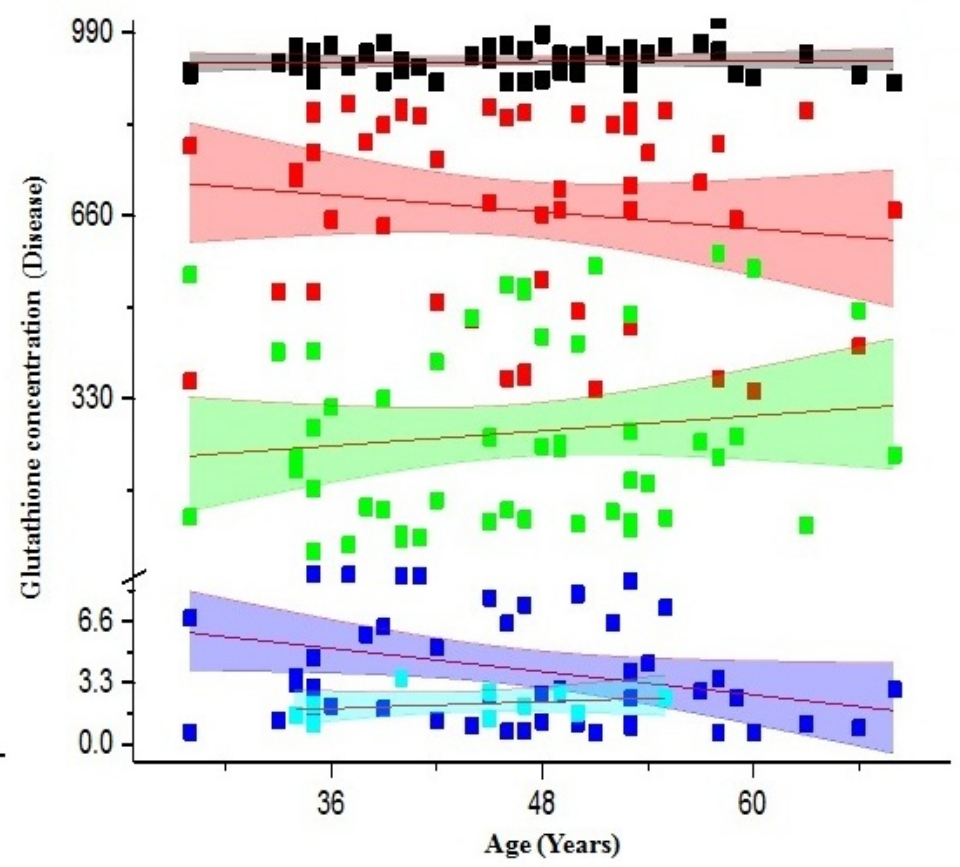

Figure 2

Correlation of endogenous level of GSH with age of breast cancer patients

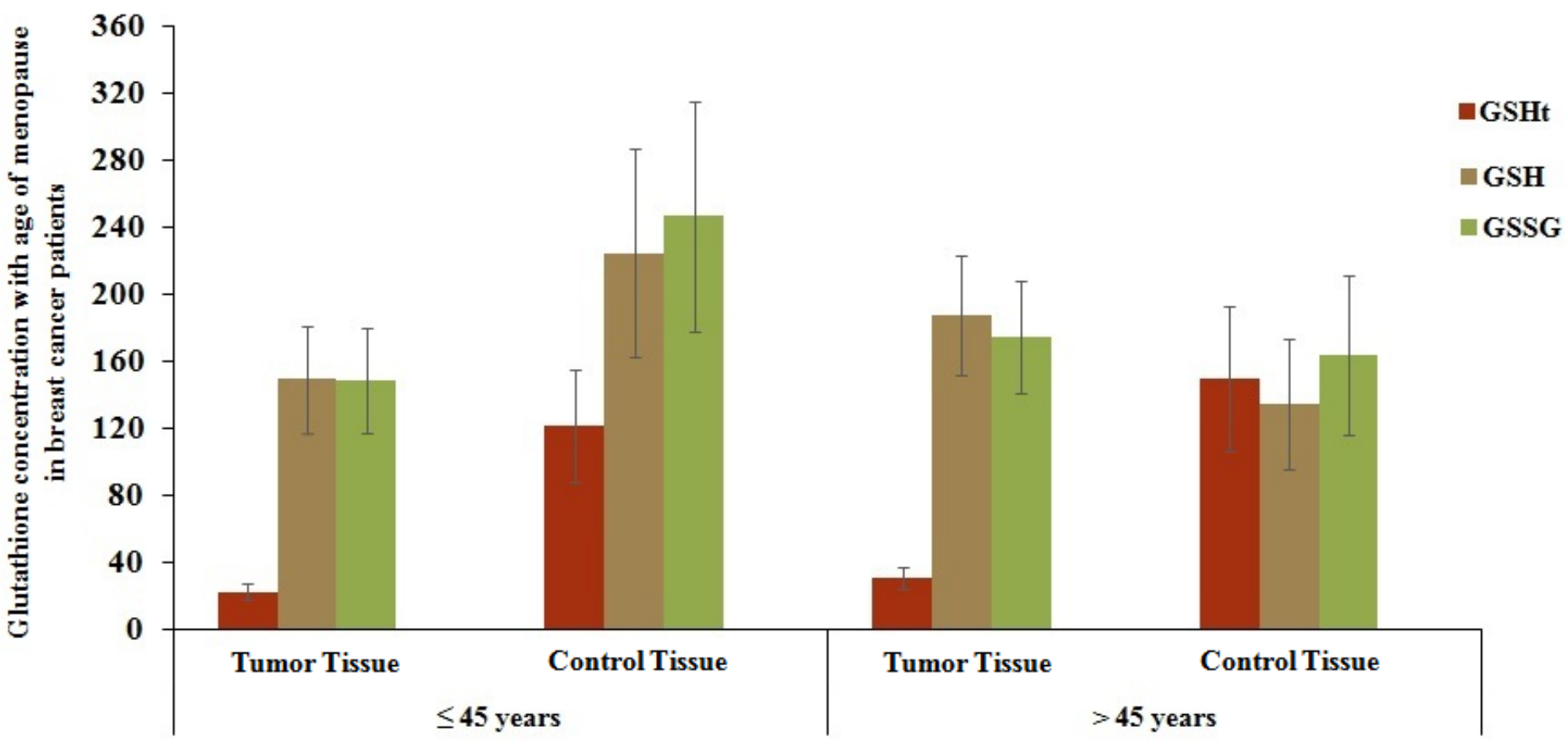

Figure 3

Determination of endogenous level of GSH associated with above and below 45 years of patients age 


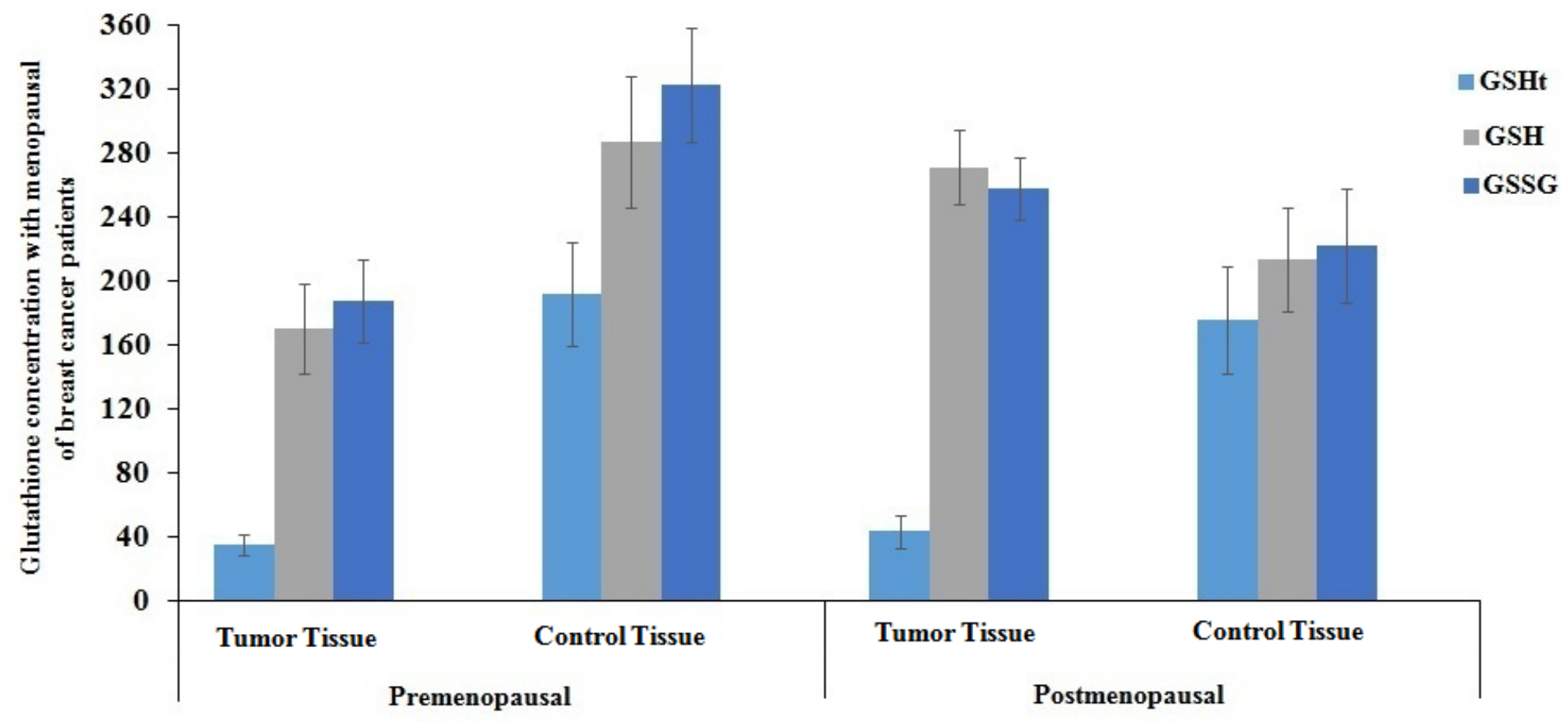

Figure 4

Association of GSH concentration with menopausal status of breast cancer patients 


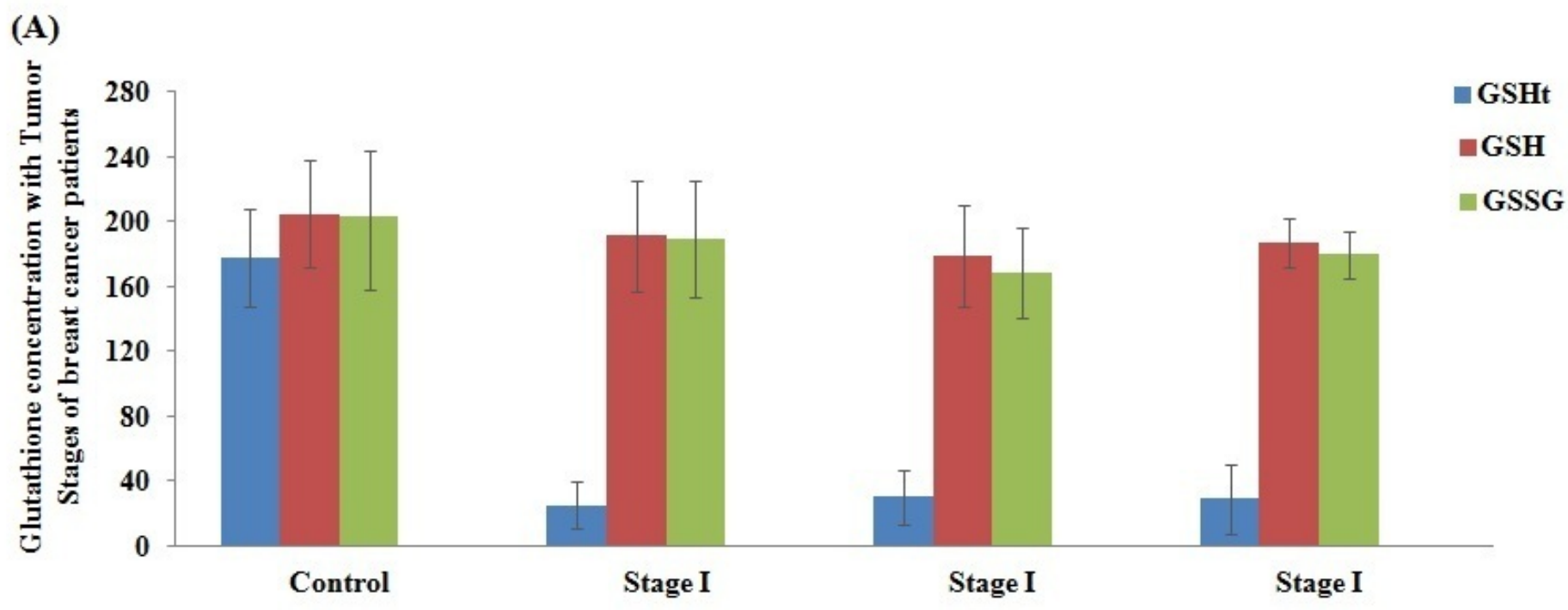

(B)

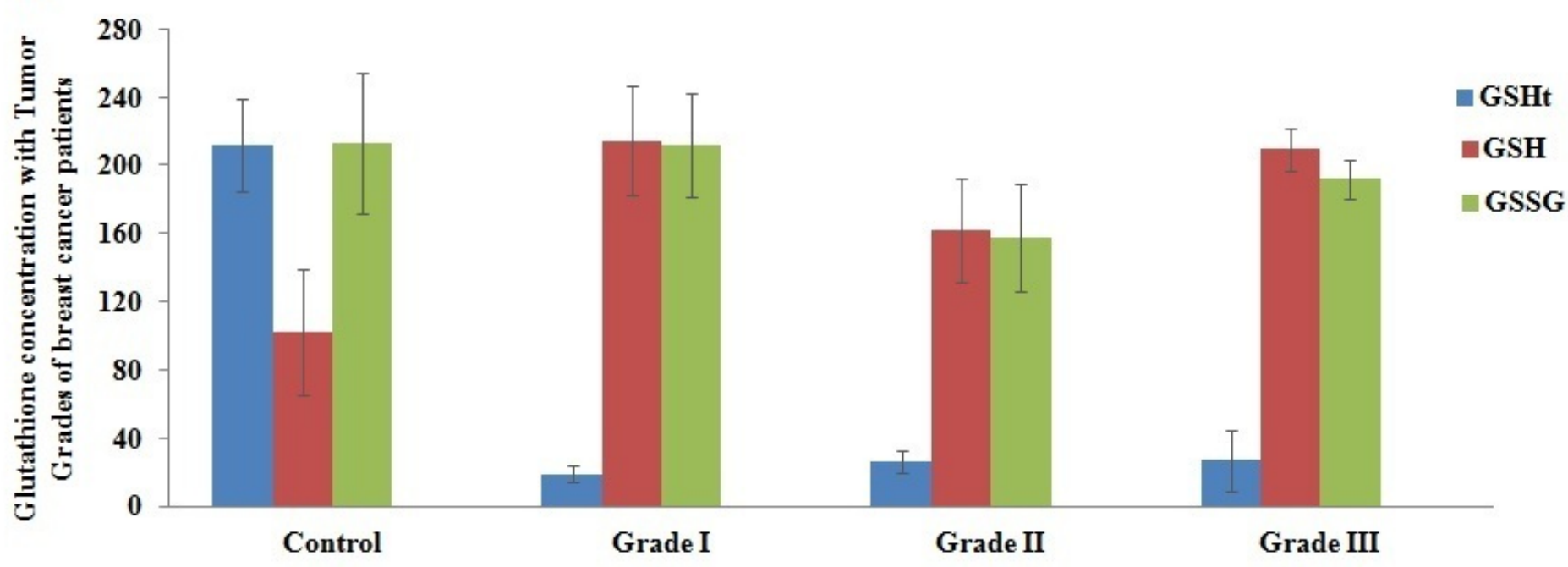

Figure 5

Association of GSH concentration with tumor stage and grade of breast cancer patients (A) Correlation of GSH level with different stages of breast cancer (B) correlation of GSH level with different grades of breast tumor tissue samples compared with ANCT samples. 

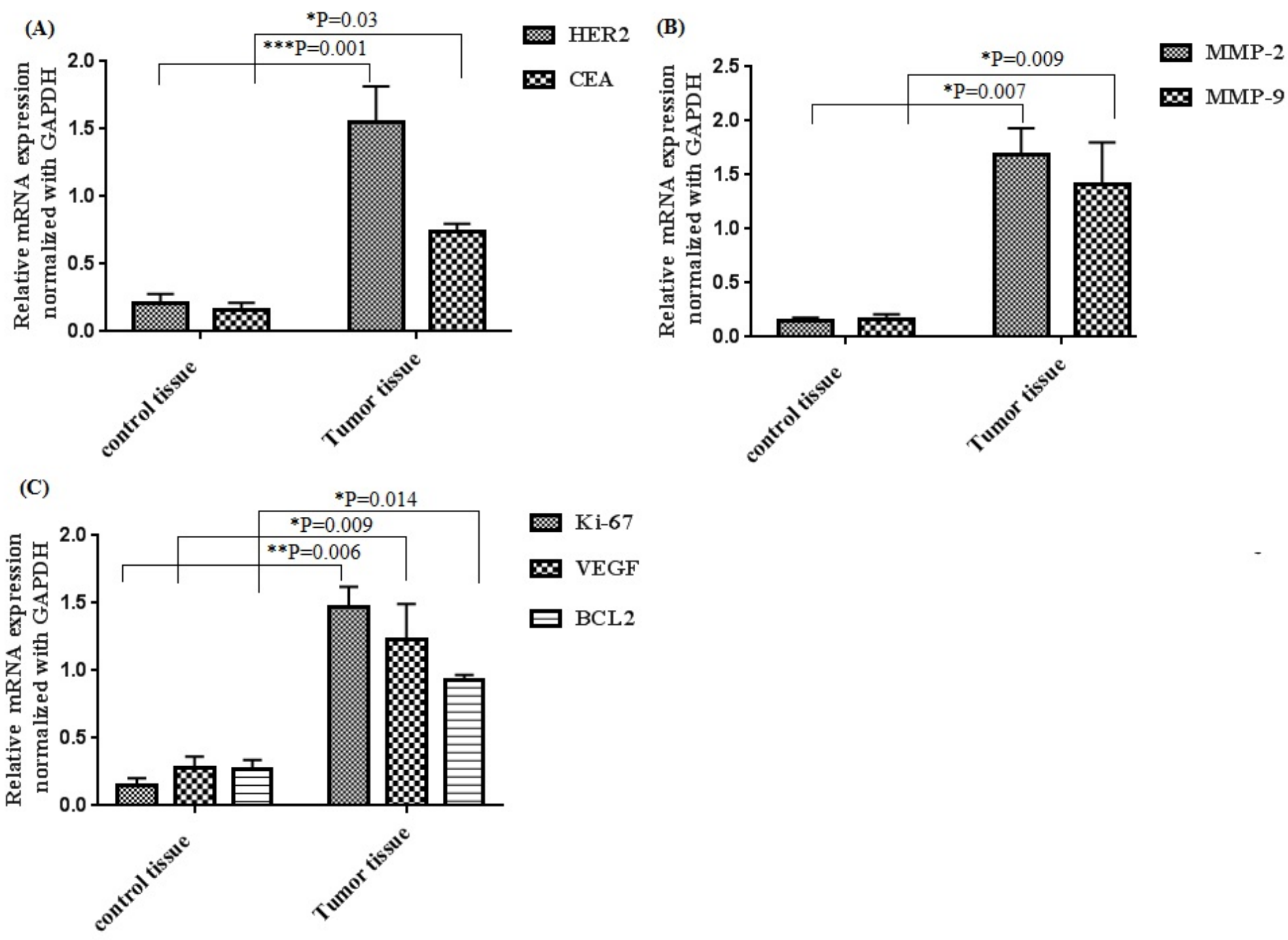

\section{Figure 6}

Determination of tumor proliferation, metastasis and invasion biomarker in breast cancer tissues (A) Relative expression of Her2 ( $* \star * p=0.001)$ and CEA ( $\left.{ }^{p} p=0.03\right)$ tumor markers, (B) Relative expression of MMP-2 ( $\left.{ }^{*} \mathrm{p}=0.007\right)$ and MMP-9 ( $\left.{ }^{*} \mathrm{p}=0.009\right)$ markers, $(\mathrm{C})$ Relative expression of Ki-67( $\left.{ }^{\star \star} \mathrm{p}=0.006\right)$, VEGF $\left({ }^{*} \mathrm{p}=0.009\right)$ and $\mathrm{BCL} 2\left({ }^{*} \mathrm{p}=0.014\right)$ markers in breast tumor tissue samples compared with ANCT samples. 
(A)

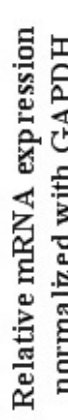

田

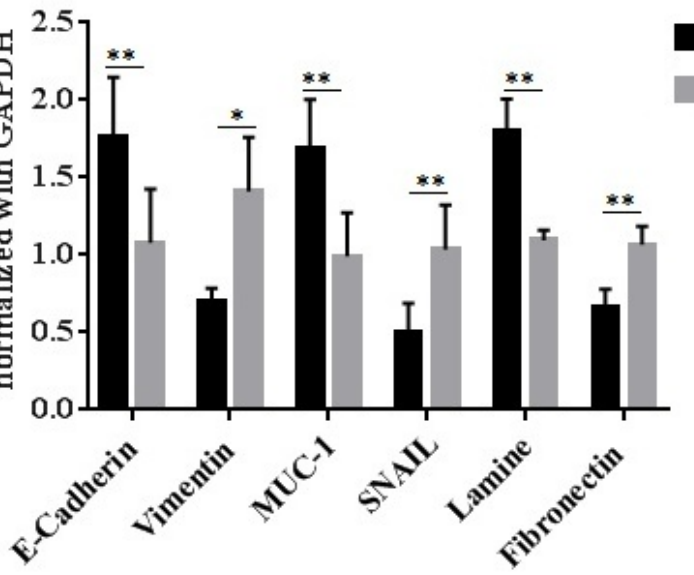

Control tissue

Tumor tissue

(B)

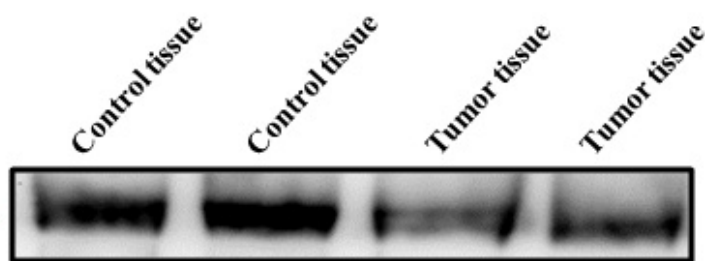

E-Cadherin

Vimentin

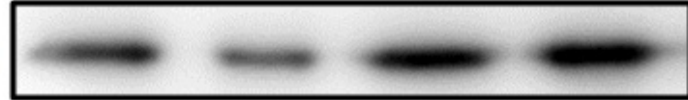

MUC-1

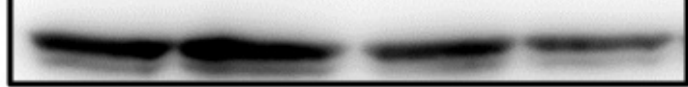

Snail

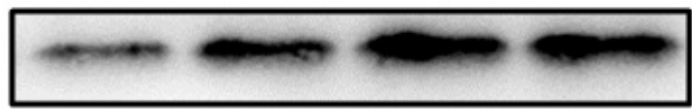

GAPDH

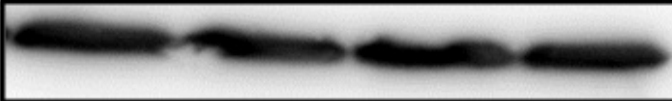

Figure 7

Aberrant expression of GSTM1 promote EMT pathway in breast cancer tissues (A) Relative mRNA expression of EMT biomarkers (B) Protein expression of EMT biomarkers in tumor tissue samples as compared to ANCT samples in breast cancer patients.

\section{Supplementary Files}

This is a list of supplementary files associated with this preprint. Click to download.

- SupplementaryTables.doc 